\title{
Influence of the regulatory protein RsmA on cellular functions in Pseudomonas aeruginosa PAO1, as revealed by transcriptome analysis
}

\author{
Elizabeth Burrowes, Christine Baysse, Claire Adams and Fergal O'Gara
}

Correspondence

Fergal O'Gara

f.ogara@ucc.ie

Received 6 July 2005

Revised 4 October 2005

Accepted 11 October 2005
BIOMERIT Research Centre, Department of Microbiology, National University of Ireland, Cork, Ireland

RsmA is a posttranscriptional regulatory protein in Pseudomonas aeruginosa that works in tandem with a small non-coding regulatory RNA molecule, RsmB (RsmZ), to regulate the expression of several virulence-related genes, including the $N$-acyl-homoserine lactone synthase genes las/ and rhll, and the hydrogen cyanide and rhamnolipid biosynthetic operons. Although these targets of direct RsmA regulation have been identified, the full impact of RsmA on cellular activities is not as yet understood. To address this issue the transcriptome profiles of $P$. aeruginosa PAO1 and an isogenic rsmA mutant were compared. Loss of RsmA altered the expression of genes involved in a variety of pathways and systems important for virulence, including iron acquisition, biosynthesis of the Pseudomonas quinolone signal (PQS), the formation of multidrug efflux pumps, and motility. Not all of these effects can be explained through the established regulatory roles of RsmA. This study thus provides both a first step towards the identification of further genes under RsmA posttranscriptional control in P. aeruginosa and a fuller understanding of the broader impact of RsmA on cellular functions.

\section{INTRODUCTION}

Pseudomonas aeruginosa is an opportunistic pathogen that causes morbidity and mortality in patients with compromised immunity, such as cystic fibrosis sufferers and burns patients (Pruitt et al., 1998; Van Delden \& Iglewski, 1998). The ability to sense aspects of the external environment and bring about timed, co-ordinate gene expression ensures that this nosocomial pathogen exploits its ecological adaptability to successfully live in the immunocompromised host. Virulence in $P$. aeruginosa is multifaceted, and involves the production of disease-causing secondary metabolites and virulence factors, nutrient scavenging, motility and biofilm formation. The GacA/RsmA/RsmB (RsmZ) signal transduction system is a conserved pathway involved in a variety of adaptive functions in $P$. aeruginosa. RsmA has numerous

\footnotetext{
Abbreviations: AHL, N-acyl homoserine lactone; C4-HSL, N-butanoyl-Lhomoserine lactone; 3-oxo-C12-HSL, N-(3-oxododecanoyl)-L-homoserine lactone; POS, Pseudomonas quinolone signal; OS, quorum sensing.

Genes with a statistically significant $(P=0.05)$, minimum twofold change in expression in the rsmA mutant compared to $P$. aeruginosa PAO1 wild-type are shown in Supplementary Table S1, genes commonly altered in expression in the $P$. aeruginosa PAK vfr mutant and the $P$. aeruginosa PAO1 rsmA mutant are shown in Supplementary Table S2, and genes commonly altered in expression in the $P$. aeruginosa PAK retS mutant and the $P$. aeruginosa PAO1 rsmA mutant are shown in Supplementary Table S3, available with the online version of this paper.
}

homologues in both Gram-negative and Gram-positive bacteria (Romeo, 1998); posttranscriptional regulatory proteins similar to RsmA have been described in Pseudomonas fluorescens CHA0 (RsmA), Escherichia coli (CsrA) and Erwinia carotovora (RsmA) (Blumer et al., 1999; Cui et al., 1995; Liu \& Romeo, 1997). CsrA is a well-documented RNA binding protein of $E$. coli that mediates posttranscriptional control of genes involved in a number of physiological pathways. CsrA represses the genes involved in glycogen biosynthesis in E. coli by binding to the $5^{\prime}$ end of the glgCAP mRNA transcript around the Shine-Dalgarno site; this prevents ribosome binding and translation and leads to rapid degradation of the mRNA (Baker et al., 2002). Positive posttranscriptional regulation has also been described in $E$. coli, in which CsrA binds to a yet-unidentified area in the $5^{\prime}$ untranslated leader sequence of the flhDC master operon that is involved in flagellum biosynthesis, thus increasing the stability of the mRNA and facilitating its translation (Wei et al., 2001).

In $P$. aeruginosa $\mathrm{PAO}$, it has been shown that the response regulator GacA has an effect on RsmA levels, as a gacA mutant expresses $30 \%$ less RsmA protein than wild-type in late-exponential phase (Pessi et al., 2001). GacA works in tandem with the transmembrane sensor kinase GacS. The GacS/GacA two-component system controls expression of virulence-related genes in response to unidentified external environmental signals. GacA also has a positive posttranscriptional effect on the $h c n A B C$ gene cluster (Pessi \& Haas, 
2001). Recently, both GacA and RsmA have been shown to modulate the levels of RsmB (RsmZ) by regulating its transcription. RsmB is the small, untranslated regulatory RNA molecule that works in tandem with RsmA (Burrowes et al., 2005; Heurlier et al., 2004). RsmB is proposed to bind and sequester RsmA, and hence limit its activity. Therefore, GacA also regulates the activity of the RsmA protein by controlling the levels of its partner molecule, RsmB. The posttranscriptional regulatory protein $\mathrm{RsmA}$ has been implicated in secondary metabolite production (Pessi et al., 2001).

Interestingly, in $P$. aeruginosa, RsmA and GacA are also involved in the regulation of quorum sensing (QS) signalling molecule synthesis. The QS system has key roles in activation and co-ordination of virulence gene expression in $P$. aeruginosa $\mathrm{PAO} 1$, in response to cell density and environmental signals. QS involves the accumulation of signal molecules, often $\mathrm{N}$-acyl homoserine lactones (AHLs), to a threshold level to activate expression of certain genes (Fuqua \& Greenberg, 1998). P. aeruginosa has two interdependent QS systems, the las and $r h l$ systems. The LasI AHL synthase produces $\mathrm{N}$-(3-oxododecanoyl)-L-homoserine lactone (3oxo-C12-HSL) and the RhlI AHL synthase produces $\mathrm{N}$ butanoyl-L-homoserine lactone (C4-HSL). These AHLs, in association with their cognate regulators, LasR and RhlR, respectively, control the transcription of a number of virulence-related genes, such as alkaline protease, and the pyocyanin and hydrogen cyanide biosynthetic genes (Gambello et al., 1993; Reimmann et al., 1997). RsmA negatively regulates lasI, rhlI and the hydrogen cyanide biosynthetic genes (Pessi et al., 2001). In contrast, RsmA has a positive posttranscriptional effect on the expression of the genes encoding the secondary metabolites lipase and rhamnolipid (Heurlier et al., 2004). GacA is also a documented hierarchical member of the QS network and has a positive transcriptional effect on the las and $r h l$ QS systems (Reimmann et al., 1997).

$P$. aeruginosa also produces a third intercellular signal molecule, 3,4-dihydroxy-2-heptylquinoline, known as the Pseudomonas quinolone signal (PQS). LasR has been shown to regulate $\mathrm{PQS}$ production, and RhlR is required for PQS activity (Pesci et al., 1999). PQS has been shown to induce expression of lasB, the gene encoding LasB elastase, and rhlI (McKnight et al., 2000; Pesci et al., 1999). PQS has also been implicated in the regulation of the secondary metabolites pyocyanin and hydrogen cyanide (Gallagher et al., 2002). Diggle et al. (2003) showed that mutants defective in PQS production or in PQS-mediated response produced substantially lower levels of $r h l$-dependent exoproducts, while C4-HSL levels were unaltered, suggesting that PQS also regulates rhl-dependent genes independently of C4-HSL. MvfR (PqsR) is a QS-associated regulator that controls the production of $\mathrm{PQS}$, pyocyanin and elastases, and is required for full $P$. aeruginosa virulence (Deziel et al., 2005; Diggle et al., 2003; Gallagher et al., 2002; Rahme et al., 1997). A recent study has revealed that LasR and RhlR indirectly regulate PQS production via MvfR, and that MvfR binds directly to the pqsABCDE operon, positively regulating its transcription (Wade et al., 2005). So far, a direct impact of RsmA or GacA on PQS synthesis has not been reported.

Recent studies have shed more light on the regulation of rsmA. Deziel et al. (2005) reported that in P. aeruginosa PA14, rsmA is posttranscriptionally regulated by MvfR. Another regulatory factor known to influence the levels of free, active RsmA is the recently identified hybrid sensor kinase/response regulator RetS (RtsM) (Goodman et al., 2004; Laskowski et al., 2004). Transcriptome profiling of the $P$. aeruginosa PAK retS mutant revealed a decrease in the expression of genes involved in type III secretion and virulence and an increase in the biosynthetic genes (the psl and pel operons) involved in the formation of the mannose- and glucose-rich exopolysaccharide component of biofilms (Goodman et al., 2004). Interestingly, these phenotypes were suppressed by transposon insertions in gacS, gacA and $r s m B$. The authors propose that, under conditions of acute infection, environmental signals favour activation of RetS, resulting in repression of the $\mathrm{GacS} / \mathrm{GacA} / \mathrm{RsmB}$ pathway. A mutation in retS results in de-repression of the levels of GacS, GacA and RsmB, and hence a decrease in the level of active RsmA. A retS mutation is, therefore, thought to cause alterations in gene expression via decreased levels of free, active RsmA (Goodman et al., 2004).

Although RsmA may be an element in signal transduction pathways with key roles in virulence, the full regulatory impact of RsmA on cellular activities in $P$. aeruginosa $\mathrm{PAO} 1$ is not understood. Here we have used transcriptome profiling to examine the global effects of an $r s m A$ mutation on gene expression in P. aeruginosa PAO1. Thus, we have identified a set of genes whose expression is most probably indirectly affected by the absence of RsmA. Validation of the transcriptome data using lac $Z$ transcriptional fusions confirmed that, amongst other targets, RsmA is involved in the modulation of $p v d S, v f r$ and pilM transcription. Analysis of these data identified a number of candidate genes, including some encoding transcriptional regulators, that may be novel targets for direct RsmA action.

\section{METHODS}

Bacterial strains, plasmids and culture conditions. The $P$. aeruginosa strains and plasmids used in this study are listed in Table 1. P. aeruginosa PAO1 strains and E. coli strains were routinely cultured at $37^{\circ} \mathrm{C}$ in Luria-Bertani (LB) broth or on LB plates. Antibiotics were used at the following concentrations: for P. aeruginosa, $70 \mu \mathrm{g}$ tetracycline $\mathrm{ml}^{-1}, 500 \mu \mathrm{g}$ chloramphenicol ml $\mathrm{ml}^{-1}, 200 \mu \mathrm{g}$ carbenicillin $\mathrm{ml}^{-1}$; for E. coli, $25 \mu \mathrm{g}$ tetracycline $\mathrm{ml}^{-1}, 30 \mu \mathrm{g}$ chloramphenicol $\mathrm{ml}^{-1}$.

DNA manipulations. Restriction-enzyme digests, ligations, transformations and agarose gel electrophoresis were performed as previously described (Sambrook et al., 1989). Small-scale plasmid DNA isolation was performed using the Qiagen Plasmid Mini kit according to the manufacturers' specifications. Restriction fragments were purified from agarose gels using the Qiagen Gel Extraction kit. PCR 
Table 1. Bacterial strains, plasmids and primers used in this study

The sequences for primers pvdSFZ, pvdSRZ, pilMFZ, pilMRZ, vfrFZ and vfrRZ include the recognition sequences (italics) for the restriction enzymes $X b a 1$ and $K p n 1 . \mathrm{Tc}^{\mathrm{R}}$, tetracycline resistant.

\begin{tabular}{|c|c|c|}
\hline $\begin{array}{l}\text { Strain, plasmid } \\
\text { or primer }\end{array}$ & $\begin{array}{l}\text { Description or sequence } \\
\qquad\left(5^{\prime} \rightarrow 3^{\prime}\right)\end{array}$ & $\begin{array}{l}\text { Reference } \\
\text { or source }\end{array}$ \\
\hline \multicolumn{3}{|l|}{ Strains } \\
\hline \multicolumn{3}{|l|}{$P$. aeruginosa } \\
\hline PAO1 & Wild-type & Holloway collection \\
\hline PAZH13 & rsmA deletion mutant of PAO1 & Pessi et al. (2001) \\
\hline MP551 & $p q s R::$ TnIS phoA/hah-Tc; derived from PAO1 & Gallagher et al. (2002) \\
\hline PAO1 phnA & phnA::Tn5, $\mathrm{Tc}^{\mathrm{R}}$ & Jacobs et al. (2003) \\
\hline \multicolumn{3}{|l|}{ Plasmids } \\
\hline pMP220 & Transcriptional reporter, $\mathrm{Tc}^{\mathrm{R}}$ & Spaink et al. (1987) \\
\hline pCU705 & rsmA in $\mathrm{pBBR} 1 \mathrm{MCS}$ & Burrowes et al. (2005) \\
\hline pLP0996 & $p q s A-l a c Z$ transcriptional fusion in pLP170 & McGrath et al. (2004) \\
\hline pCUB24 & pilM-lacZ transcriptional fusion in pMP220 & This study \\
\hline pCUB25 & $p v d S-l a c Z$ transcriptional fusion in pMP220 & This study \\
\hline pCUB26 & $v f r-l a c Z$ transcriptional fusion in pMP220 & This study \\
\hline pRTLF-1 & rpoS-lacZ translational fusion in pMP77 & Bertani et al. (2003) \\
\hline \multicolumn{3}{|l|}{ Primers } \\
\hline proCF & CAGGCCGGGCAGTTGCTGTC & Savli et al. (2003) \\
\hline proCR & GGTCAGGCGCGAGGCTGTCT & Savli et al. (2003) \\
\hline piluF & TGCGCCTGATGGTGGAAAAG & This study \\
\hline pilUR & TTCCGGGAGCTTCAGCTCTT & This study \\
\hline pilMF & GCTGGTTTTCCAGTTCATCCT & This study \\
\hline pilMR & GACATCAGCTCGACCTCGG & This study \\
\hline oprNF & GTTCAGTCTGCTGGCGCTC & This study \\
\hline oprNR & TTGCTGGCCCTTGCCGATG & This study \\
\hline pvdSF & CCGTACGATCCTGGTGAAGA & This study \\
\hline pvdSR & GATCACCACGTTCAGGCCTT & This study \\
\hline gloA2F & TGCGAATCCTGCACAGCATG & This study \\
\hline gloA2R & ATCACGCTGCGTCCGTGCT & This study \\
\hline pilMFZ & CCCGGGGGTACCCGACCAATTCCCTATTAGCG & This study \\
\hline pilMRZ & CCCGGGTCTAGAGAGTTAAGCGAATAGGACAGG & This study \\
\hline pvdSFZ & CCCGGGGGTACCGGAACGGATAACAACGGCAAA & This study \\
\hline pvdSRZ & CCCGGGTCTAGATGGAAATCACCTTGCTGCGG & This study \\
\hline vfrFZ & CCCGGGGGTACCCGGGATCACAGTCCTGATAG & This study \\
\hline vfrRZ & CCCGGGTCTAGACATGCCCGAGTCCCGAAAGA & This study \\
\hline
\end{tabular}

products were purified using the High Pure PCR Product Purification kit (Boehringer). Plasmids were introduced into Pseudomonas strains via electroporation.

Microarray sample preparation and analysis. Duplicate cultures of $P$. aeruginosa $\mathrm{PAO} 1$ wild-type and the $r s m A$ mutant were grown in $\mathrm{LB}$ broth to an $\mathrm{OD}_{600}$ of $0 \cdot 8$, and $4 \mathrm{ml}$ of each culture was treated with RNAprotect bacterial reagent (Qiagen), according to the manufacturers' instructions. Cells were harvested by centrifugation and stored at $-80^{\circ} \mathrm{C}$. Total RNA was extracted using the RNeasy Midi RNA Isolation kit (Qiagen) according to the manufacturers' instructions. The RNA was treated with RQ1 RNase-free DNase (Promega) for $1 \mathrm{~h}$ at $37^{\circ} \mathrm{C}$ and purified using the Clean-Up protocol of the RNeasy Midi RNA Isolation kit according to the manufacturers' instructions. The RNA was precipitated with ethanol and the RNA pellet was resuspended in sterile distilled $\mathrm{H}_{2} \mathrm{O}$ to a final volume of $20 \mu \mathrm{l}$; the integrity of the RNA was checked on an agarose gel and quantified using a spectrophotometer. cDNA was synthesized according to the Affymetrix Expression Analysis Protocol guide; spike RNA transcripts from Bacillus subtilis genes were included in the reaction. The cDNA was treated with $2 \mu \mathrm{g}$ DNasefree RNase (Roche) in a final volume of $100 \mu \mathrm{l}$ for $1 \mathrm{~h}$ at $37^{\circ} \mathrm{C}$, purified using the QIAquick PCR Purification kit (Qiagen) and eluted in $45 \mu \mathrm{l}$ sterile distilled $\mathrm{H}_{2} \mathrm{O}$. The cDNA was fragmented using RQ1 RNase-free DNase for $1 \mathrm{~h}$ at $37^{\circ} \mathrm{C}$, and biotin-labelled using the BioArray Terminal Labelling kit with biotin-ddUTP (Enzo Life Sciences). The cDNA was snap-frozen on dry ice and analysed at the MRC Geneservice (Cambridge, England) on Affymetrix GeneChip $P$. aeruginosa Genome arrays. A Student's $t$ test was carried out on the data generated from the microarray analysis using GeneDirector software (BioDiscovery) to give a list of genes with altered expression greater than twofold and a $P$ value $\leqslant 0 \cdot 05$.

Semi-quantitative RT-PCR analysis. To confirm the microarray data, semi-quantitative RT-PCR analysis was carried out. RNA was isolated from cultures of PAO1 wild-type and the rsmA mutant grown to an $\mathrm{OD}_{600}$ of 0.8 in $\mathrm{LB}$ broth, and converted to cDNA using the procedure already described. The primers used to amplify 
the genes gloA2, pilM, pvdS, oprN, pilU and proC are listed in Table 1. PCRs were carried out using PCR Master Mix (Promega) and $50 \mathrm{ng}$ cDNA template for 20-25 cycles. The constitutively expressed control gene proC (Savli et al., 2003) was used as the internal control to verify the absence of significant variation in cDNA levels in all samples.

Construction and analysis of transcriptional fusions. The promoters and start sites of $p v d S$, pilM and $v f r$ were amplified from $P$. aeruginosa PAO1 DNA using primers listed in Table 1. The primers (pvdSFZ, pvdSRZ, pilMFZ, pilMRZ, vfrFZ, vfrRZ) include the recognition sequences (Table 1 , italics) for the restriction enzymes $X b a 1$ and Kpnl. The resultant PCR products were digested using $X b a 1$ or $K p n 1$, PCR-purified, and cloned into the reporter fusion pMP220 (Spaink et al., 1987), upstream of the promoterless lac $Z$ gene. $\beta$-Galactosidase activity was measured by standard methods (Sambrook et al., 1989). Due to the clumping phenotype of the rsmA mutant, total protein was extracted from $2 \mathrm{ml}$ of cells at each time-point analysed, and this number was substituted for $\mathrm{OD}_{600}$ to calculate $\beta$-galactosidase activity.

Western blot analysis. Total protein was extracted from strains of $P$. aeruginosa $\mathrm{PAO} 1$, grown in $\mathrm{LB}$ broth to an $\mathrm{OD}_{600}$ of 0.8 and quantified using the Bio-Rad Protein Assay Dye Reagent. Proteins and All Blue Precision Plus Protein standard (Bio-Rad) were electrophoresed on $5 \%$ polyacrylamide SDS stacking gels and $15 \%$ polyacrylamide SDS resolving gels at $130 \mathrm{~V}$ using the Mini-PROTEAN II cell (Bio-Rad). Proteins were either stained with Coomassie brilliant blue (BDH Chemicals) or transferred to a nitrocellulose membrane using a Mini Trans-Blot cell (Bio-Rad). The membrane was blocked with $5 \%$ non-fat dried milk, $0 \cdot 1 \%(\mathrm{v} / \mathrm{v})$ Tween 20 in Tris-buffered saline (TBS) for $1 \mathrm{~h}$, washed three times in TBS/Tween and incubated with anti-PvdS monoclonal antibodies (received from Professor M. Vasil, University of Colorado) for $2 \mathrm{~h}$ at room temperature. The membrane was washed three times in TBS/Tween and incubated with anti-mouse secondary antibodies (Dakocytomation) for $1 \mathrm{~h}$. Detection was performed using an ECL + kit (Amersham Pharmacia Biotech) and chemiluminescent film (Kodak).

Pyocyanin quantification. Production of the phenazine pyocyanin by $P$. aeruginosa $\mathrm{PAO} 1$ wild-type and the $\operatorname{rsmA}$ mutant was measured as described elsewhere (Essar et al., 1990).

Motility assay. Swimming was analysed on tryptone swim plates ( $1 \%$, w/v, tryptone, $0.5 \%, \mathrm{w} / \mathrm{v}, \mathrm{NaCl}, 0 \cdot 3 \%, \mathrm{w} / \mathrm{v}$, agar), which were inoculated using a sterile toothpick with bacteria from colonies grown overnight on LB agar at $37^{\circ} \mathrm{C}$. The plates were incubated at $37^{\circ} \mathrm{C}$, and motility was assessed qualitatively by examining the circular turbid zone formed by the bacterial cells migrating away from the point of inoculation.

Adhesion assay. Adhesion on polyvinyl chloride (PVC) was tested using non-coated 24-well tissue-culture flat-well plates (Sarstedt). LB $(1 \mathrm{ml})$ was added to each well and inoculated to a starting $\mathrm{OD}_{600}$ of $0 \cdot 05$. The plates were incubated at $37^{\circ} \mathrm{C}$ without agitation for $12 \mathrm{~h}$. The wells were rinsed with water and the bacterial film was stained with $1 \%$ crystal violet. Six replicates were performed per sample. The wells were then washed with $1 \mathrm{ml} 95 \%$ ethanol and the $\mathrm{OD}_{570}$ of a $1: 100$ dilution in sterile distilled $\mathrm{H}_{2} \mathrm{O}$ was obtained.

PQS extraction and analysis. Cultures of $P$. aeruginosa $\mathrm{PAO} 1$ wild-type, PAZH13 (rsmA mutant), PAO1 pCU705 (rsmA overexpressing strain), the $P$. aeruginosa pqsR mutant (Gallagher et al., 2002) and the $P$. aeruginosa phnA mutant (Jacobs et al., 2003) were grown to early stationary phase in LB broth. Culture $(3 \mathrm{ml})$ was extracted with $3 \mathrm{ml}$ acidified ethyl acetate, the solvent removed by rotary evaporation and the extract resuspended in $1: 1$ acidified ethyl acetate/acetonitrile. Silica gel $60 \mathrm{~F}_{254}$ aluminium TLC plates (Merck) were activated by soaking for $15 \mathrm{~min}$ in $5 \% \mathrm{KH}_{2} \mathrm{PO}_{4}$, wrapping in tin foil and heating to $100^{\circ} \mathrm{C}$ for $1 \mathrm{~h}$. Samples, PQS standard (Diggle et al., 2003) and anthranilate standard (Sigma) were separated on the TLC plates using 95:5 dichloromethane/ methanol in a solvent chamber. PQS appeared as a blue/purple spot under UV light.

\section{RESULTS}

\section{Microarray analysis of $P$. aeruginosa PA01 wild-type and the rsmA mutant}

It has been shown previously that RsmA levels peak during late-exponential phase, which is the time-point at which RsmA maximally affects target gene expression (Pessi et al., 2001). Therefore, in order to study the impact of the loss of rsmA on gene expression, duplicate transcriptome analyses were carried out on $P$. aeruginosa PAO1 wild-type and PAZH13, the rsmA mutant, grown to late-exponential phase in LB broth at $37^{\circ} \mathrm{C}$. Under these conditions, the growth rate and final $\mathrm{OD}_{600}$ of PAO1 wild-type and the rsmA mutant were identical (data not shown). The transcriptome analysis was carried out using four Affymetrix GeneChip P. aeruginosa Genome arrays (see Methods). A Student's $t$ test was carried out on the comparative data generated from the microarrays using GeneDirector software (BioDiscovery) to give a list of genes with a statistically significant $(P \leqslant 0 \cdot 05)$, minimum twofold, change in expression (Table 2 and Supplementary Table S1).

To confirm and validate the microarray data, semiquantitative RT-PCR was carried out on five genes (pilM, pilU, oprN, pvdS and gloA2). These genes were chosen for RT-PCR analysis because their fold change in expression in the $r$ smA mutant compared to PAO1 wild-type varied across a relatively broad range $(+1173$ for gloA2 expression and -3 for pilM expression) and they were physiologically relevant candidates for further analysis. The constitutively expressed gene proC (Savli et al., 2003), which encodes a pyrroline-5-carboxylate reductase, and whose expression was not altered in the $r s m A$ mutant, was used as an internal control in these reactions. The RT-PCR analyses correlated with the microarray alterations in gene expression: pilM and pilU showed reduced expression, and $g l o A 2, p v d S$ and $o p r N$ showed increased expression in the rsmA mutant compared to wild-type (Fig. 1).

Of the 5570 genes present on the array, 506 genes (9\%) showed significantly altered expression in the rsmA mutant compared to PAO1. Most altered genes $(n=365$ or $6 \cdot 5 \%)$ were decreased in expression in the rsmA mutant compared to PAO1. All genes of known function, with altered expression in the rsmA mutant compared to PAO1, are listed in Table 2. A large number of altered genes $(n=222$ or $44 \%)$ were categorized as Class 4 or of unknown function according to the Pseudomonas website classification system (http:// www.pseudomonas.com; Supplementary Table S1). Where possible, genes of altered expression were classified into functional groups (Table 2). The expression of RNA complementary to 29 intergenic regions was also significantly 
Table 2. Genes of altered expression in $P$. aeruginosa rsmA compared to $P$. aeruginosa PAO1 wild-type, arranged into functional groups

Positive values represent genes increased in expression in the rsmA mutant compared to PAO1; negative values represent genes decreased in expression in the rsmA mutant compared to PAO1. PA numbers and annotations are from the Pseudomonas website (http://www. pseudomonas.com).

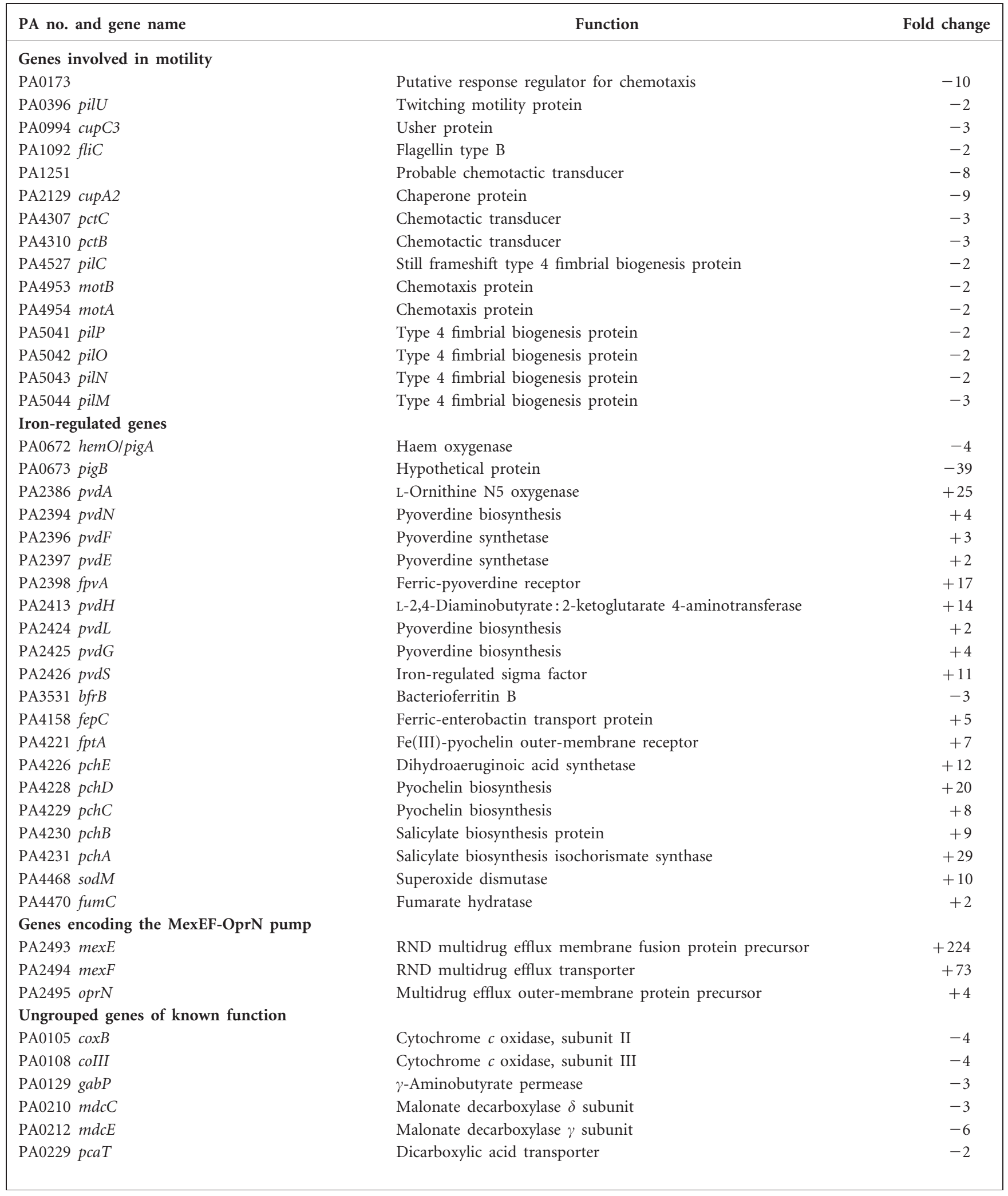


Table 2. cont.

\begin{tabular}{|c|c|c|}
\hline PA no. and gene name & Function & Fold change \\
\hline PA0235 pcaK & 4-Hydroxybenzoate transporter & -3 \\
\hline PA0652 vfr & Transcriptional regulator & -2 \\
\hline PA0706 cat & Chloramphenicol acetyltransferase & -23 \\
\hline PA0710 gloA2 & Lactoylglutathione lyase & +1173 \\
\hline PA0723 соaB & Coat protein $\mathrm{B}$ of bacteriophage Pf1 & +5 \\
\hline PA0843 plcR & Phospholipase accessory protein precursor & -2 \\
\hline PA0844 plcH & Haemolytic phospholipase C precursor & +3 \\
\hline PA0905 rsmA & Regulator of secondary metabolites & -192 \\
\hline PA0958 oprD & $\begin{array}{l}\text { Basic amino acid, basic peptide and imipenem } \\
\text { outer-membrane porin OprD precursor }\end{array}$ & -4 \\
\hline PA1001 phnA & Anthranilate synthase component I & -3 \\
\hline PA1148 toxA & Exotoxin A precursor & -3 \\
\hline PA1151 imm2 & Pyocin S2 immunity protein & -2 \\
\hline PA1173 napB & Cytochrome $c$-type protein NapB precursor & -2 \\
\hline PA1247 aprE & Alkaline protease secretion protein & -3 \\
\hline PA1338 ggt & $\gamma$-Glutamyltranspeptidase precursor & -2 \\
\hline PA1393 cysC & Adenosine $5^{\prime}$-phosphosulfate (APS) kinase & -11 \\
\hline PA1409 aphA & Acetylpolyamine aminohydrolase & -6 \\
\hline PA1635 $k d p C$ & Potassium-transporting ATPase, C chain & -5 \\
\hline PA1691 pscT & Translocation protein in type III secretion & -2 \\
\hline PA1695 pscP & Translocation protein in type III secretion & -11 \\
\hline PA1698 popN & Type III secretion outer-membrane protein PopN precursor & -4 \\
\hline PA1758 pabB & para-Aminobenzoate synthase component I & +4 \\
\hline PA1770 ppsA & Phosphoenolpyruvate synthase & +2 \\
\hline PA1781 nirB & Assimilatory nitrite reductase large subunit & -3 \\
\hline PA1947 rbsA & Ribose transport protein & -3 \\
\hline PA1949 rbsR & Ribose operon repressor & -2 \\
\hline PA1983 exaB & Cytochrome $c_{550}$ & -3 \\
\hline PA2007 maiA & Maleylacetoacetate isomerase & -4 \\
\hline PA2009 hmgA & Homogentisate 1,2-dioxygenase & -4 \\
\hline PA2064 pcoB & Copper resistance protein B precursor & -3 \\
\hline PA2247 bkdA1 & 2-Oxoisovalerate dehydrogenase ( $\alpha$ subunit) & -2 \\
\hline PA2248 bkdA2 & 2-Oxoisovalerate dehydrogenase ( $\beta$ subunit) & -3 \\
\hline PA2337 $m t l R$ & Transcriptional regulator & -2 \\
\hline PA2509 catB & Muconate cycloisomerase I & -3 \\
\hline PA2926 hisP & Histidine transport protein & -5 \\
\hline PA3540 algD & GDP-mannose 6-dehydrogenase & -3 \\
\hline PA3543 algK & Alginate biosynthetic protein & -3 \\
\hline PA3544 algE & Alginate production outer-membrane protein & -5 \\
\hline PA3549 algJ & Alginate $o$-acetyltransferase & -3 \\
\hline PA3603 dgkA & Diacylglycerol kinase & +3 \\
\hline PA4110 ampC & $\beta$-Lactamase precursor & -2 \\
\hline PA4127 hpcG & 2-oxo-hept-3-ene-1,7-dioate hydratase & -4 \\
\hline PA4138 tyrS & Tyrosyl-tRNA synthetase & -2 \\
\hline PA4205 mеxG & Hypothetical protein & -3 \\
\hline PA4210 phzA1 & Probable phenazine biosynthetic protein & -3 \\
\hline PA4356 xenB & Xenobiotic reductase & +8 \\
\hline PA4522 ampD & $\beta$-Lactamase expression regulator & -2 \\
\hline PA4855 purD & Phosphoribosylamine-glycine ligase & +2 \\
\hline PA5243 hemB & $\delta$-Aminolevulinic acid dehydratase & -2 \\
\hline PA5355 glcD & Glycolate oxidase subunit & -4 \\
\hline PA5361 phoR & Two-component sensor & -5 \\
\hline PA5501 znuB & Permease of $\mathrm{ABC}$ zinc transporter & -2 \\
\hline
\end{tabular}




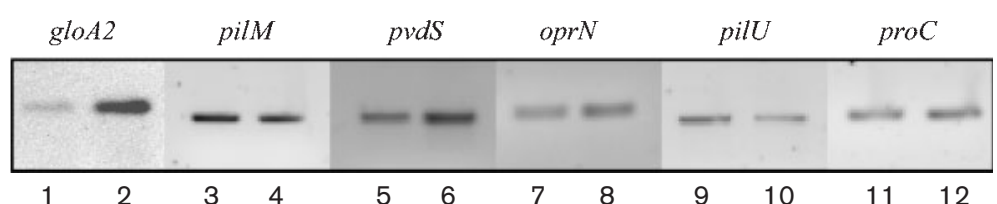

Fig. 1. Semi-quantitative RT-PCR analysis of gloA2, pilM, pvdS, oprN, pilU and proC in $P$. aeruginosa PAO1 wild-type (lanes 1 , $3,5,7,9$ and 11) and the $\operatorname{rsm} A$ mutant (lanes 2, 4, 6, 8, 10 and 12). altered by inactivation of $\operatorname{rsm} A$. This may indicate yet-unidentified ORFs or the presence of untranslated regulatory RNA molecules. Indeed, RsmA has been shown previously to positively regulate the transcription of the gene encoding its cognate regulatory untranslated RNA, RsmB (Burrowes et al., 2005; Heurlier et al., 2004). However, the $r p o S / f d x A$ intergenic region, in which $r s m B$ is located, is not represented on the Affymetrix $P$. aeruginosa PAO1 GeneChip.

\section{Functional categories of genes with increased expression in the rsmA mutant compared to PA01 wild-type}

Iron acquisition. The expression of genes involved in iron acquisition was increased in the rsmA mutant compared to PAO1 wild-type. The expression of a number of pvd and pch genes, which direct the biosynthesis of the siderophores pyoverdine and pyochelin, respectively, was increased in the $r s m A$ mutant compared to wild-type. In addition, expression of $f p v A$, the ferric-pyoverdine receptor, and $f p t A$, the ferric-pyochelin receptor, was increased in the rsmA mutant compared to PAO1 wild-type. Interestingly, the microarray analysis revealed that expression of the gene encoding the iron-regulated sigma factor $\operatorname{PvdS}$ was increased 11-fold in the rsmA mutant compared to wild-type. The fact that PvdS levels were increased in the rsmA mutant may explain the increase in expression of the pvd genes (Beare et al., 2003; Visca et al., 2002) but not the pch operon. Transcription of pchABCDE and $f p t A$ is activated by the AraC-like regulator PchR in the presence of pyochelin and repressed in the absence of pyochelin (Heinrichs \& Poole, 1996). Although expression of the pch genes was increased in the rsmA mutant, the levels of $p c h R$ transcript were not altered. Therefore, the increase in pch transcript levels may reflect a change in mRNA stability rather than in transcription rate, or the implementation of an alternate regulator. To confirm that the observed increase in $p v d S$ levels in the rsmA mutant compared to $\mathrm{PAO} 1$ wild-type was due to a change in transcription rate, a pvdS-lacZ transcriptional fusion (pCUB25) was constructed in the fusion vector pMP220. Transcription of $p v d S$ was increased in the rsmA mutant compared to PAO1 wild-type at all time-points analysed (Fig. 2a). To ascertain if this increase in transcription corresponded to an increase in PvdS protein levels, Western blot analysis was carried out on total protein isolated from PAO1 wild-type and from the rsmA mutant grown to an $\mathrm{OD}_{600}$ of 0.8 in LB broth. The $21 \cdot 2 \mathrm{kDa}$ PvdS protein was detected with an anti-PvdS antibody via chemiluminescence, and PvdS levels were found to be higher in the rsmA mutant compared to PAO1 wild-type, albeit by a moderate increase (Fig. 3). Band intensities were quantified using GeneTools software (version 3.05.03, Syngene). The mean band intensity in the rsmA mutant sample, as a percentage of mean band intensity of the wild-type sample, was $155 \pm 20 \%(n=3)$.

Both PvdS and Vfr have been shown previously to positively regulate toxA expression (Lamont et al., 2002; West et al., 1994). Although PvdS levels were increased in the rsmA mutant, toxA showed a $2 \cdot 8$-fold decrease in expression in the rsmA mutant. This may be due to the drop in $v f r$ levels in the $r s m A$ mutant (see below), suggesting that under the conditions of this experiment, the loss of the positive influence of Vfr on toxA transcription is more potent than the increase in PvdS levels on toxA transcription.

The expression of genes involved in resistance to oxidative stress, namely sodM and fumC (Hassett et al., 1997) was also increased. This is consistent with an increase in iron uptake, which generates reactive hydroxyl radicals via the Fenton reaction.

Antibiotic resistance. Loss of $r s m A$ resulted in increased expression of the genes encoding the MexEF-OprN pump. In $P$. aeruginosa, the MexEF-OprN pump removes numerous antibiotics and PQS from the cell (Poole, 2001). Several proteins have been implicated in the positive regulation of expression of this Mex system, including MexT, an activator of MexEF-OprN (Maseda et al., 2000). In addition, spontaneous $n f x C$ mutants show an increase in expression of the MexEF-OprN pump (Kohler et al., 2001). Examination of MexT protein levels in the rsmA mutant showed that they were not altered (data not shown). However, levels of mexS mRNA, which encodes a transcriptional repressor of MexEF-OprN, (Sobel et al., 2005) were increased fourfold in the $r s m A$ mutant compared to PAO1. These results reflect the complexity of Mex pump regulation.

\section{Functional categories of genes with decreased expression in the rsmA mutant compared to PA01 wild-type}

POS biosynthesis. PQS is a third signal molecule in addition to C4-HSL and 3-oxo-C12-HSL produced by $P$. aeruginosa, and $\mathrm{PQS}$ production occurs at the onset of stationary phase (Diggle et al., 2003; Pesci et al., 1999). Microarray analysis revealed that $p h n A$ expression was decreased by $2 \cdot 6$-fold in the rsmA mutant compared to PAO1 wild-type. The phnAB operon encodes an anthranilate synthase, which converts chorismate to anthranilate, a precursor of PQS and pyocyanin. PhnA is essential for 
(a) pvdS-lacZ

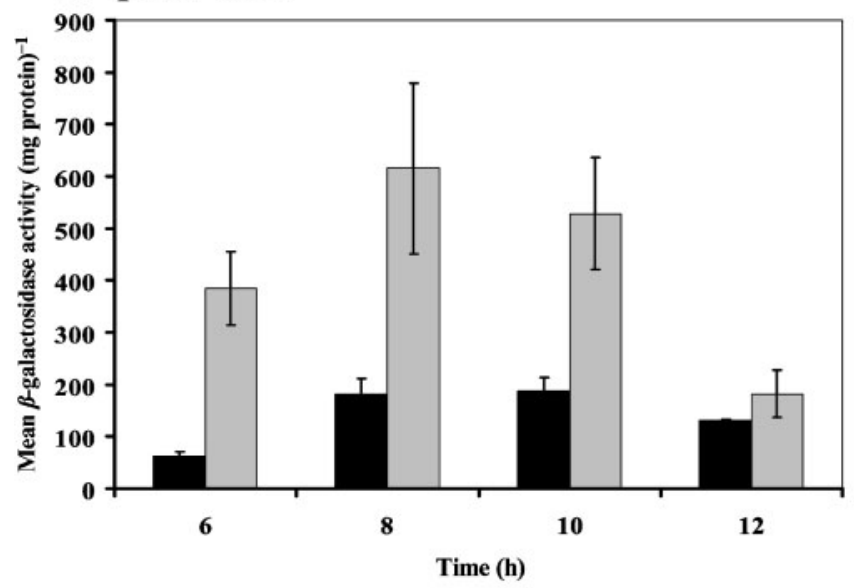

(c) pilM-lac Z

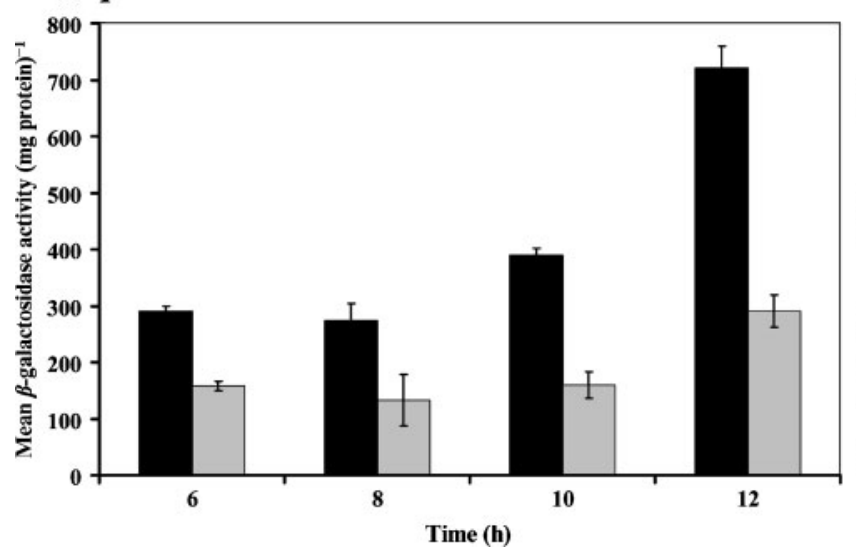

(b) pqsA-lacZ

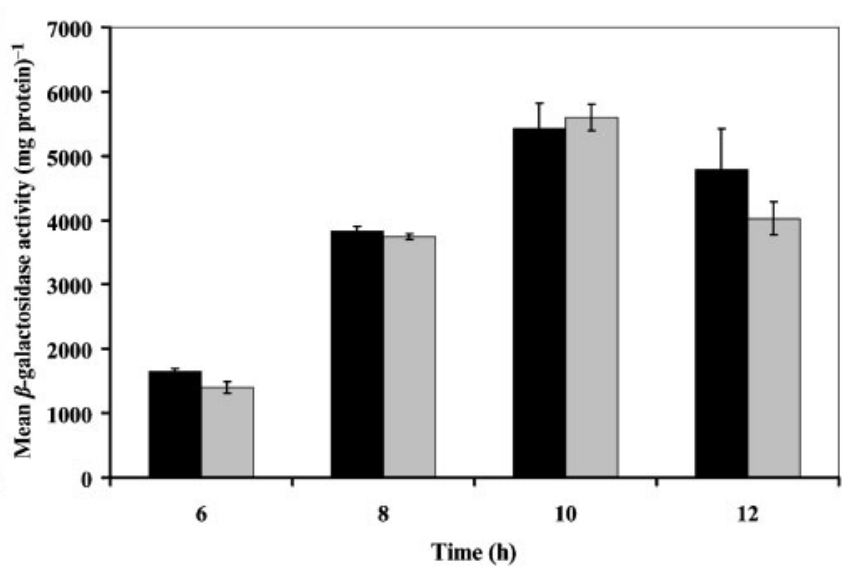

(d) vfr-lac Z

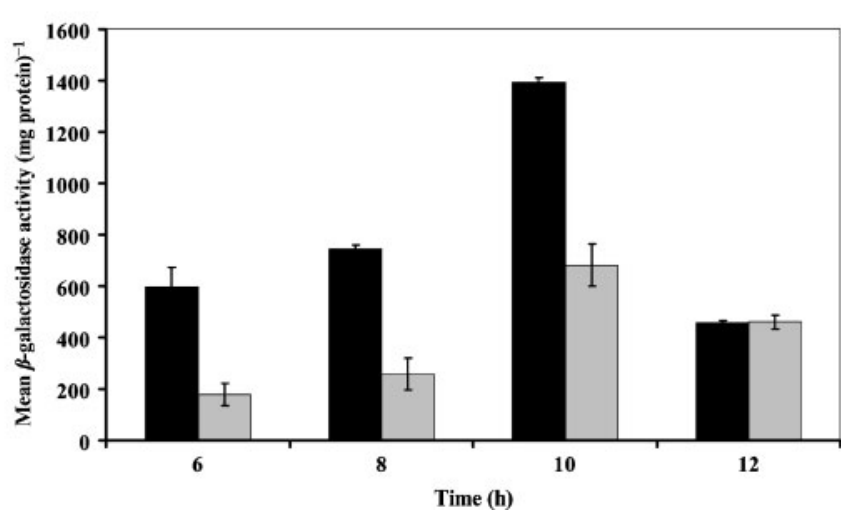

Fig. 2. Kinetics of expression of (a) pvdS (pCUB25), (b) pqsA (pLP0996), (c) pilM (pCUB24) and (d) vfr (pCUB26) transcriptional reporter lacZ fusions in $P$. aeruginosa PAO1 (black bars) and in the rsmA mutant (grey bars). Values are the mean and standard deviation of triplicate measurements.

PQS production in P. aeruginosa, as a phnA mutant does not make PQS (Gallagher et al., 2002). However, levels of the pqsABCDE transcripts, which encode the PQS biosynthetic enzymes, did not appear to be altered in the comparative microarray analyses. To validate a transcript recorded as 'no change' on the microarray, confirmatory analysis was performed with a pqsA-lac $Z$ transcriptional fusion (pLP0996) (Fig. 2b). As expected, there was no significant difference in the transcription of the pqsABCDE operon in PAO1 wild-type and the rsmA mutant. To examine the impact of the decrease in expression of $p h n A$ on PQS levels in the rsmA mutant, PQS extractions were carried out on supernatants of PAO1, the rsmA mutant, PAO1 pCU705 $\left(r s m A^{++}\right)$, MP551 (PAO1 pqsR) and PAO1 phnA grown to stationary phase $\left(\mathrm{OD}_{600}\right.$ of $\left.3 \cdot 0\right)$ in LB broth. Fig. 4 shows that the rsmA mutant produced less PQS than PAO1 wild-type in stationary phase and that PAO1 overexpressing rsmA produced more PQS than PAO1 wild-type. This was accompanied by a parallel reduction in the intensity of the upper band, namely compound B; this compound has not yet been identified, but its levels have been found to parallel PQS levels in certain $P$. aeruginosa strains, including PAO1 (Collier et al., 2002). The large spot present in the TLC analysis of the $r s m A$, $p q s R$ and $p h n A$ mutants is a precursor of $\mathrm{PQS}$, namely anthranilate (Baysse et al., 2005). As expected, neither PQS nor compound $\mathrm{B}$ was present in the $p q s R$ mutant, as this gene product is essential for their synthesis. In addition, it was interesting to note that the PQS profile of the $r s m A$ mutant was similar to that of the phnA mutant, supporting the transcriptome finding that expression of $p h n A$ was reduced in the $r s m A$ mutant.

The PhnAB system also provides the anthranilate required for the biosynthesis of the phenazine pyocyanin (Gallagher et al., 2002); levels of pyocyanin in stationary-phase cultures of PAO1 and the rsmA mutant were examined. This revealed that loss of RsmA substantially reduced the production of pyocyanin when the bacteria were cultured in LB; PAO1 wild-type produced $4 \cdot 2 \mu \mathrm{g} \mathrm{ml}^{-1}( \pm 0 \cdot 1, n=3)$ of pyocyanin, while the $r s m A$ mutant only produced $0.3 \mu \mathrm{g} \mathrm{ml}^{-1}$ $( \pm 0 \cdot 05, n=3)$ of pyocyanin in stationary phase $\left(\mathrm{OD}_{600}\right.$ of 


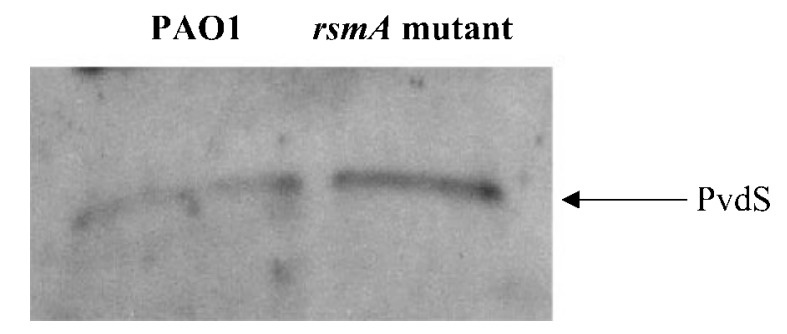

Fig. 3. Western blot analysis of PvdS levels in total protein extracted from $P$. aeruginosa PAO1 and the rsmA mutant grown to an $\mathrm{OD}_{600}$ of 0.8 in LB broth.

4). However, under the same experimental conditions in glycerol/alanine medium, which was used as a control, the rsmA mutant showed an increase in production of pyocyanin compared to wild-type: PAO1 wild-type produced $0 \cdot 23 \mu \mathrm{g} \mathrm{ml}^{-1}( \pm 0 \cdot 03, n=3)$ of pyocyanin, while the $r s m A$ mutant produced $3 \cdot 27 \mu \mathrm{g} \mathrm{ml}^{-1}( \pm 0 \cdot 01, n=3)$ of pyocyanin, which is in accordance with the previous observation by Pessi et al. (2001). The result obtained in LB is in agreement with the observed accumulation of the metabolic precursor anthranilate and the drop in levels of PQS in the $r s m A$ mutant. Indeed, PQS has been shown to be required for phenazine gene expression in PAO1 (Diggle et al., 2003). Interestingly, expression of $p h z A 1$, the first gene in one of the pyocyanin biosynthetic operons (Mavrodi et al., 2001), was decreased by approximately threefold in the $r s m A$ mutant compared to PAO1 wild-type. The other genes in the operon were recorded as 'absent' for the rsmA mutant, which is most likely due to mRNA or cDNA degradation during sample preparation for microarray analysis.

Motility. RsmA positively regulates fimbrial biosynthetic genes in P. aeruginosa PAO1. Transcriptome analysis revealed that expression of genes involved in the formation of type IV pili, fimbriae and chemotaxis was decreased in the rsmA mutant compared to PAO1 wild-type (Table 2). Expression of the pilMNOPQ operon and pilU, which encode proteins of unknown function that are thought to be involved in the biosynthesis of pili (Mattick et al., 1996), was decreased in the rsmA mutant compared to wild-type. Expression of a third pil gene, pilC, was also decreased in the rsmA mutant compared to PAO1. To examine the altered transcription of the pilMNOPQ operon, a pilM-lacZ transcriptional fusion was constructed in pMP220 (pCUB24) and analysed over time in PAO1 and the rsmA mutant (Fig. 2c). This confirmed that transcription of the pilMNOPQ operon was decreased in the mutant throughout all phases of growth. Depending on the carbon source used, type IV pili are not always necessary for the initial attachment of $P$. aeruginosa PAO1, but they are always necessary for normal biofilm development, irrespective of growth medium (Klausen et al., 2003). Under the conditions used for this transcriptome study (i.e. LB broth), the $P$. aeruginosa $r s m A$ mutant $\left(\mathrm{OD}_{570}\right.$ $0 \cdot 20 \pm 0 \cdot 06, n=5)$ was significantly impaired for biofilm formation on PVC compared to wild-type $\left(\mathrm{OD}_{570} 0 \cdot 34 \pm\right.$ $0 \cdot 05, n=5)$ (Fig. 5a).

The transcriptome analysis also showed that components of the chaperone/usher (cup) pathways in P. aeruginosa were down-regulated in the rsmA mutant compared to PAO1 (Table 2). The cup genes are thought to be involved in the assembly of novel $P$. aeruginosa fimbrial structures that are different from type IV pili; the cupA gene cluster is required for cell adhesion and initiation of biofilm formation (Vallet et al., 2001). In the rsmA mutant, expression of cupA2, which encodes a fimbrial chaperone protein, was down ninefold and expression of сupC3, which encodes an usher protein, was down approximately threefold compared to PAO1. Interestingly, mutations in cupA2 have been shown to cause cell clumping in P. aeruginosa PAK (Vallet et al., 2001), so it is possible that the clumping phenotype observed for the rsmA mutant (Heurlier et al., 2004) is caused by the drop in cupA2 levels. The expression of a number of genes involved in chemotaxis was also decreased in the rsmA mutant compared to PAO1. PA0173, which encodes a putative response regulator for chemotaxis, was decreased tenfold, and expression of PA1251, which encodes a probable chemotaxis

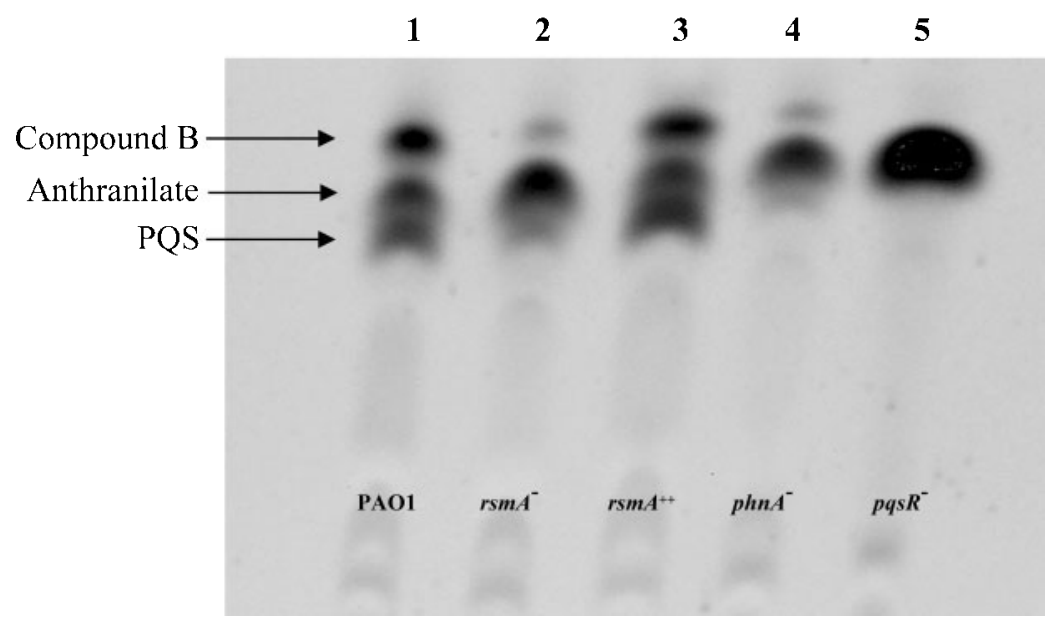

Fig. 4. POS extracts from $P$. aeruginosa PAO1 wild-type (lane 1), the $\operatorname{rsm} A$ mutant (lane 2), PAO1 pCU705 (rsm $A^{++}$) (lane 3), $P$. aeruginosa phnA (lane 4 ) and $P$. aeruginosa pqs $R$ (lane 5) grown to an $\mathrm{OD}_{600}$ of 0.8 in LB broth. 
(a)

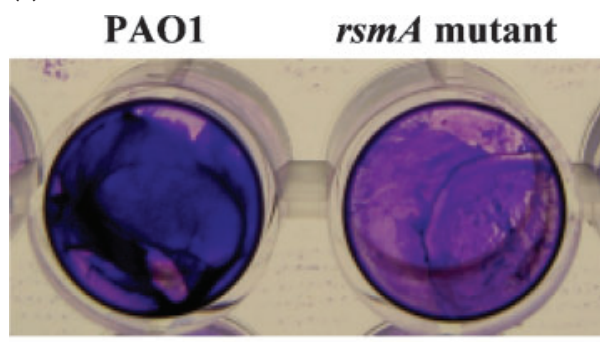

(b)

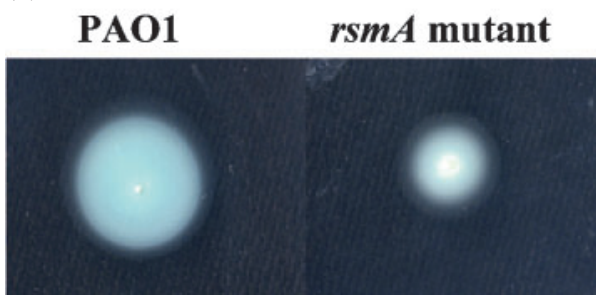

Fig. 5. (a) Biofilm formation on PVC by $P$. aeruginosa PAO1 wild-type and the $\operatorname{rsm} A$ mutant grown in LB, as revealed by crystal violet staining; (b) Swimming motility of $P$. aeruginosa PAO1 wild-type and the $P$. aeruginosa $\operatorname{rsm} A$ mutant.

transducer, was down eightfold in the rsmA mutant compared to wild-type. The decreased expression of genes involved in chemotaxis may explain the drop in swimming motility in the rsmA mutant compared to PAO1 (Fig. 5b). The mot $A$ and $m o t B$ genes encode proteins that make up the stator or stationary component of the flagellar motor (Toutain et al., 2005). Expression of motA and $\operatorname{mot} B$ was down twofold in the rsmA mutant compared to PAO1 (Table 2). From these data, it is evident that RsmA is involved in a complex regulatory cascade that controls the expression of genes involved in motility in $P$. aeruginosa.

Vfr transcription. Microarray analysis revealed that expression of $v f r$ was decreased twofold in the $r s m A$ mutant compared to PAO1 wild-type. Vfr is a homologue of the E. coli cAMP receptor protein Crp. Crp is activated by binding cAMP and then binds to a specific sequence in the promoter of Crp-controlled genes. In P. aeruginosa, Vfr has been implicated in the regulation of twitching motility, QS, pyocyanin production, repression of flagellar biosynthesis and the expression of numerous proteins secreted by the type II pathway, including exotoxin A (Albus et al., 1997; Beatson et al., 2002; Dasgupta et al., 2002; West et al., 1994). As mentioned previously, toxA expression was decreased approximately $2 \cdot 8$-fold in the rsmA mutant compared to PAO1. The expression of regA, lasR and fleQ, other known Vfr targets, was not altered in the rsmA mutant. However, the transcription of regA, lasR and fleQ is also controlled by numerous other regulators (Dasgupta \& Ramphal, 2001; Dasgupta et al., 2003; Hunt et al., 2002; Juhas et al., 2005), which could compensate for the drop in $v f r$ levels. To confirm the novel impact of RsmA on Vfr levels, a vfr-lacZ transcriptional fusion (pCUB26) was constructed and analysed in PAO1 and the rsmA mutant (Fig. 2d). This analysis showed that $v f r$ expression peaked after $10 \mathrm{~h}$ of growth, corresponding to late-exponential/early stationary phase, in both the wildtype and the $r s m A$ mutant. Expression of $v f r$ was decreased in the rsmA mutant compared to PAO1 throughout the growth cycle until late stationary phase, in which $v f r$ transcription was the same in both backgrounds.

Iron storage. Finally, the expression of $b f r B$, the gene encoding the bacterioferritin iron-storage protein, was down-regulated in the rsmA mutant compared to PAO1, which is in agreement with the increase in expression of genes involved in siderophore-mediated iron uptake (Table 2). Therefore, although grown in iron-rich LB medium, the rsmA mutant behaved partly as though it was cultured in iron-deplete conditions.

\section{DISCUSSION}

RsmA exerts its effect at the posttranscriptional level. Commonplace methods for identifying targets of posttranscriptional regulators include gene-by-gene transcriptional and translational fusion analyses, RNA binding studies, and proteomics. However, to provide a comprehensive list of genes whose expression is influenced by RsmA, we chose the somewhat unconventional approach of transcriptome profiling to examine the global effects of an $r s m A$ mutation on gene expression and to identify novel functions influenced by RsmA in $P$. aeruginosa PAO1. As regulatory networks in $P$. aeruginosa have been intensively documented, changes in gene expression enable identification of known transcriptional regulators as potential direct targets for RsmA.

A notable feature of this transcriptome analysis was the farreaching consequences of an $r s m A$ mutation, emphasizing the role of RsmA as a global regulator. RsmA is known to indirectly control the transcription of numerous genes through regulation of the QS network (Pessi et al., 2001). Hence, some of the observed alterations in the transcriptome profile of the rsmA mutant may be due to the direct effect of the rsmA mutation on the QS network, since RsmA negatively regulates lasI and rhlI (Pessi et al., 2001). For example, the increase in 3-oxo-C12-HSL in the rsmA mutant (Pessi et al., 2001) may contribute to the increase in expression of the pyoverdine biosynthetic genes (Stintzi et al., 1998; Whiteley et al., 1999) and sodM (Hassett et al., 1999), both regulated by the QS system. However, although inactivation of $r s m A$ affects AHL synthesis, this does not appear to have a dramatic effect on expression of all QSregulated genes. Moreover, alteration in AHL levels in the rsmA mutant cannot explain all of the changes in gene expression brought about by the loss of RsmA, so further explanations must be considered. An important feature of this study, which cannot be overlooked, is that microarray 
experiments measure mRNA steady-state levels, which are influenced by transcription rate and mRNA stability and decay. The only known method of RsmA-mediated regulation of gene expression occurs through alteration of mRNA stability and subsequent translation. However, the known influence of RsmA on the stability of certain mRNAs may not be sufficient to explain the extent of changes observed in the comparative transcriptome profile. Therefore, given that several phenotypes were greatly affected by inactivation of RsmA, we believe that RsmA posttranscriptionally regulates one or more transcriptional regulators, which in turn affect the transcription of a number of genes.

RsmA is involved in the expression of $p v d S$ and siderophore biosynthetic genes. A recently identified link between the QS network and iron acquisition in P. aeruginosa is VqsR (Juhas et al., 2004). VqsR is a regulator of the QS network in $P$. aeruginosa $\mathrm{TB}$, and has been shown to regulate genes involved in iron acquisition. vqs $R$ mutants have reduced C4HSL and 3-oxo-C12-HSL production, reduced expression of both the $p h n A B$ and $p q s A B C D E$ genes, and reduced expression of $p v d S$, along with reduced transcription of other genes in the pyoverdine and pyochelin biosynthetic loci (Juhas et al., 2004). As both RsmA and VqsR influence AHL production, and the expression of phnA and the genes involved in iron acquisition, it is possible to consider a potential link between these two global regulators, which has not been previously recognized. Clearly more work needs to be carried out to unravel the involvement of RsmA in the complex, highly regulated iron acquisition and storage system in $P$. aeruginosa.

During late-exponential and stationary phase, cultures of the $r s m A$ mutant produced less PQS than PAO1 wild-type. The QS network has been implicated in the regulation of PQS production: the las system positively controls the transcription of $p q s A$, while the $r h l$ system negatively controls the transcription of $p q s A$ (McGrath et al., 2004), via the impact of both systems on pqsR expression (Wade et al., 2005). However, as neither $p q s R$ nor $p q s A$ transcription was altered in the $r s m A$ mutant, it is unlikely that the increased AHL levels (Pessi et al., 2001) influence the decrease in PQS production in the rsmA mutant. Therefore, another explanation for this drop in PQS levels was sought. In P. aeruginosa, there are two anthranilate synthase systems: $\mathrm{PhnA}$ and $\mathrm{PhnB}$ (anthranilate synthase II) and TrpE and TrpG (anthranilate synthetase I) (Essar et al., 1990). Both systems have different functions, in that anthranilate synthetase I converts chorismate to the anthranilate used in tryptophan biosynthesis, while anthranilate synthase II converts chorismate to the anthranilate used in phenazine biosynthesis (Essar et al., 1990). It has been suggested recently that anthranilate produced by anthranilate synthetase I (the Trp pathway) is not available for PQS biosynthesis (Aendekerk et al., 2005). As $\operatorname{trp} G$ and $\operatorname{trp} E$ expression was not altered in the rsmA mutant compared to PAO1, the anthranilate present in the rsmA mutant extract is likely to be an accumulation of anthranilate formed via the anthranilate synthetase I system. As phnA transcription was decreased by approximately threefold in the rsmA mutant compared to PAO1 wild-type, while pqsABCDE levels were not altered, it is possible that the anthranilate required for $\mathrm{PQS}$ formation is not available in the rsmA mutant, hence the reduction in PQS levels. Interestingly, loss of RsmA brought about a dramatic drop in levels of the phenazine pyocyanin. This finding is consistent with the decrease in phnA expression in the $r s m A$ mutant compared to PAO1 wild-type, since a phnA mutant has been shown elsewhere to have reduced pyocyanin levels (Essar et al., 1990). In addition, the decrease in expression of phzAl in the rsmA mutant may contribute to the decrease in pyocyanin production. Other studies have shown that loss of RsmA results in an increase in pyocyanin production compared to $P$. aeruginosa $\mathrm{PAO} 1$ wild-type, when the bacteria are cultured in glycerol/alanine broth (Pessi et al., 2001). However, our data show that this is not the case when the bacteria are cultured in LB broth. It is possible that the different carbon sources used in the two experiments alter pyocyanin production, via the activation of the PhnAB versus the TrpEG pathway, which would explain the discrepancy in the results.

We have shown that RsmA affects expression of $v f r$, encoding a homologue of the E. coli cAMP receptor protein. Comparison of the transcriptome profiles of the $P$. aeruginosa PAK $v f r$ mutant (Wolfgang et al., 2003) and the P. aeruginosa PAO1 rsmA mutant showed that 19 genes and one intergenic region were commonly altered in expression (Supplementary Table S2). The influence of RsmA on the expression of this subset of genes may therefore be through regulation of $v f r$, particularly with respect to the pil genes involved in the biosynthesis of pili. Vfr-null mutants have been shown to exhibit severely reduced twitching motility with barely detectable levels of type IV pili, as the mutant is defective in type IV pilus assembly (Beatson et al., 2002). Beatson et al. (2002) propose that it is most likely that Vfr acts as a transcriptional regulator of a gene(s) encoding functional elements of type IV pili or signal transduction pathways that control twitching motility. If this is indeed the case, the drop in $v f r$ levels in the rsmA mutant could explain the reduced expression of genes involved in pilus biosynthesis. Another candidate target of direct RsmA control is the regulatory protein MvaT. Recently, MvaT has been shown to be a negative regulator of the transcription of cupA genes and, to a lesser extent, of сирB and сирC expression (Vallet et al., 2004). Expression of cupA2 and cupC3 was decreased in the rsmA mutant compared to PAO1 wild-type. As a regulator of the cup genes, it is therefore possible that RsmA has posttranscriptional control over mvaT. Since mvaT transcript levels were not altered in the $r s m A$ mutant, as revealed by the transcriptome assay, it is likely that RsmA affects the translation of $m v a T$ rather than the stability of its mRNA.

Since the retS mutant is thought to alter gene expression in part by controlling the level of free, active RsmA (Goodman et al., 2004), we assessed the similarities between the 
transcriptome profiles of the $P$. aeruginosa PAK retS mutant and the $P$. aeruginosa PAO1 rsmA mutant, and genes of altered expression in both backgrounds were compared. This revealed that 53 altered genes were common to both mutants (Supplementary Table S3), hence strengthening the proposal put forward by Goodman et al. (2004) that RsmA is part of the RetS regulon.

Recent studies conducted by Heeb et al. (2005) have shown that in P. fluorescens CHA0, RsmA posttranscriptionally regulates rpoS. The stationary-phase sigma factor RpoS has been implicated in the regulation of numerous functions in $P$. aeruginosa, including QS-regulated processes (Schuster et al., 2004). To assess the posttranscriptional impact of the loss of RsmA on rpoS, an rpoS-lacZ translational fusion was analysed in $P$. aeruginosa PAO1 wild-type and the $r s m A$ mutant over time. However, there was no difference in the translation of rpoS between the two strains (data not shown). Therefore, in contrast to $P$. fluorescens CHA0, $P$. aeruginosa PAO1 RsmA does not posttranscriptionally regulate rpoS, under the experimental conditions outlined.

Interestingly, the gene with the highest fold increase in expression (1173-fold) (Table 2) in the rsmA mutant compared to PAO1 wild-type was gloA2, which encodes an enzyme involved in the breakdown of the toxic electrophile methylglyoxal, a by-product of glycolysis. This suggests that, like CsrA in E. coli (Romeo, 1998), RsmA posttranscriptionally regulates genes involved in carbon metabolism in P. aeruginosa.

In conclusion, this study has revealed the impact of RsmA on gene expression and the regulation of key cellular processes in $P$. aeruginosa. In addition, evidence is provided suggesting that RsmA may function as a downstream effector of hierarchical regulatory proteins, such as RetS and MvfR.

\section{ACKNOWLEDGEMENTS}

The authors would like to thank Pat Higgins for technical assistance and Dr Max Dow for helpful discussions. Our thanks to Professor P. Williams for supplying us with $P$. aeruginosa PAZH13, Professor E. Pesci for supplying us with pLP0996, Professor V. Venturi for supplying us with pRTLF-1 and Professor M. Vasil for supplying us with antibodies raised against PvdS. This work was supported in part by grants awarded by the Higher Education Authority of Ireland (PRTLI programmes to F. O. G.), The Science Foundation of Ireland (SFI 02/IN.1/B1261, 04/BR/B0597 to F.O.G.), the European Commission (QLK3-CT-2000-31759, QLTK3-CT-2001-0010, QLK5CT-2002-0091 to F. O. G.) and the Health Research Board (RP76/2001 to F. O. G., RP/2004/145 to F. O. G.).

\section{REFERENCES}

Aendekerk, S., Diggle, S. P., Song, Z., Hoiby, N., Cornelis, P., Williams, P. \& Camara, M. (2005). The MexGHI-OpmD multidrug efflux pump controls growth, antibiotic susceptibility and virulence in Pseudomonas aeruginosa via 4-quinolone-dependent cell-to-cell communication. Microbiology 151, 1113-1125.
Albus, A. M., Pesci, E. C., Runyen-Janecky, L. J., West, S. E. \& Iglewski, B. H. (1997). Vfr controls quorum sensing in Pseudomonas aeruginosa. J Bacteriol 179, 3928-3935.

Baker, C. S., Morozov, I., Suzuki, K., Romeo, T. \& Babitzke, P. (2002). CsrA regulates glycogen biosynthesis by preventing translation of $g \operatorname{lgC}$ in Escherichia coli. Mol Microbiol 44, 1599-1610.

Baysse, C., Cullinane, M., Dénervaud, V., Burrowes, E., Dow, J. M., Morrissey, J. P., Tam, L., Trevors, J. T. \& O'Gara, F. (2005). Modulation of quorum sensing in Pseudomonas aeruginosa through alteration of membrane properties. Microbiology 151, 2529-2542.

Beare, P. A., For, R. J., Martin, L. W. \& Lamont, I. L. (2003). Siderophore-mediated cell signalling in Pseudomonas aeruginosa: divergent pathways regulate virulence factor production and siderophore receptor synthesis. Mol Microbiol 47, 195-207.

Beatson, S. A., Whitchurch, C. B., Sargent, J. L., Levesque, R. C. \& Mattick, J. S. (2002). Differential regulation of twitching motility and elastase production by Vfr in Pseudomonas aeruginosa. J Bacteriol 184, 3605-3613.

Bertani, I., Sevo, M., Kojic, M. \& Venturi, V. (2003). Role of GacA, LasI, RhlI, Ppk, PsrA, Vfr and ClpXP in the regulation of the stationary-phase sigma factor rpoS/RpoS in Pseudomonas. Arch Microbiol 180, 264-271.

Blumer, C., Heeb, S., Pessi, G. \& Haas, D. (1999). Global GacAsteered control of cyanide and exoprotease production in Pseudomonas fluorescens involves specific ribosome binding sites. Proc Natl Acad Sci U S A 96, 14073-14078.

Burrowes, E., Abbas, A., O'Neill, A., Adams, C. \& O'Gara, F. (2005). Characterisation of the regulatory RNA RsmB from Pseudomonas aeruginosa PAO1. Res Microbiol 156, 7-16.

Collier, D. N., Anderson, L., McKnight, S. L., Noah, T. L., Knowles, M., Boucher, R., Schwab, U., Gilligan, P. \& Pesci, E. C. (2002). A bacterial cell-to-cell signal in the lungs of cystic fibrosis patients. FEMS Microbiol Lett 215, 41-46.

Cui, Y., Chatterjee, A., Liu, Y., Dumenyo, C. K. \& Chatterjee, A. K. (1995). Identification of a global repressor gene, rsmA, of Erwinia carotovora subsp. carotovora that controls extracellular enzymes, $\mathrm{N}$-(3-oxohexanoyl)-L-homoserine lactone, and pathogenicity in softrotting Erwinia spp. J Bacteriol 177, 5108-5115.

Dasgupta, N. \& Ramphal, R. (2001). Interaction of the antiactivator FleN with the transcriptional activator FleQ regulates flagellar number in Pseudomonas aeruginosa. J Bacteriol 183, 6636-6644.

Dasgupta, N., Ferrell, E. P., Kanack, K. J., West, S. E. \& Ramphal, R. (2002). fleQ, the gene encoding the major flagellar regulator of Pseudomonas aeruginosa, is $\sigma^{70}$ dependent and is downregulated by Vfr, a homolog of Escherichia coli cyclic AMP receptor protein. J Bacteriol 184, 5240-5250.

Dasgupta, N., Wolfgang, M. C., Goodman, A. L., Arora, S. K., Jyot, J., Lory, S. \& Ramphal, R. (2003). A four-tiered transcriptional regulatory circuit controls flagellar biogenesis in Pseudomonas aeruginosa. Mol Microbiol 50, 809-824.

Deziel, E., Gopalan, S., Tampakaki, A. P., Lepine, F., Padfield, K. E., Saucier, M., Xiao, G. \& Rahme, L. G. (2005). The contribution of MvfR to Pseudomonas aeruginosa pathogenesis and quorum sensing circuitry regulation: multiple quorum sensing-regulated genes are modulated without affecting lasRI, rhlRI or the production of $\mathrm{N}$ acyl-L-homoserine lactones. Mol Microbiol 55, 998-1014.

Diggle, S. P., Winzer, K., Chhabra, S. R., Worrall, K. E., Camara, M. \& Williams, P. (2003). The Pseudomonas aeruginosa quinolone signal molecule overcomes the cell density-dependency of the quorum sensing hierarchy, regulates rhl-dependent genes at the onset of stationary phase and can be produced in the absence of LasR. Mol Microbiol 50, 29-43. 
Essar, D. W., Eberly, L., Hadero, A. \& Crawford, I. P. (1990). Identification and characterization of genes for a second anthranilate synthase in Pseudomonas aeruginosa: interchangeability of the two anthranilate synthases and evolutionary implications. J Bacteriol 172, 884-900.

Fuqua, C. \& Greenberg, E. P. (1998). Self-perception in bacteria: quorum sensing with acylated homoserine lactones. Curr Opin Microbiol 1, 183-189.

Gallagher, L. A., McKnight, S. L., Kuznetsova, M. S., Pesci, E. C. \& Manoil, C. (2002). Functions required for extracellular quinolone signalling by Pseudomonas aeruginosa. J Bacteriol 184, 6472-6480.

Gambello, M. J., Kaye, S. \& Iglewski, B. H. (1993). LasR of Pseudomonas aeruginosa is a transcriptional activator of the alkaline protease gene $(a p r)$ and an enhancer of exotoxin A expression. Infect Immun 61, 1180-1184.

Goodman, A. L., Kulasekara, B., Rietsch, A., Boyd, D., Smith, R. S. \& Lory, S. (2004). A signalling network reciprocally regulates genes associated with acute infection and chronic persistence in Pseudomonas aeruginosa. Dev Cell 7, 745-754.

Hassett, D. J., Howell, M. L., Ochsner, U. A., Vasil, M. L., Johnson, Z. \& Dean, G. E. (1997). An operon containing fumC and $\operatorname{sodA}$ encoding fumarase $\mathrm{C}$ and manganese superoxide dismutase is controlled by the ferric uptake regulator in Pseudomonas aeruginosa: fur mutants produce elevated alginate levels. J Bacteriol 179, 1452-1459.

Hassett, D. J., Ma, J. F., Elkins, J. G. \& 10 other authors (1999). Quorum sensing in Pseudomonas aeruginosa controls expression of catalase and superoxide dismutase genes and mediates biofilm susceptibility to hydrogen peroxide. Mol Microbiol 34, 1082-1093.

Heeb, S., Valverde, C., Gigot-Bonnefoy, C. \& Haas, D. (2005). Role of the stress sigma factor RpoS in GacA/RsmA-controlled secondary metabolism and resistance to oxidative stress in Pseudomonas fluorescens CHA0. FEMS Microbiol Lett 243, 251-258.

Heinrichs, D. E. \& Poole, K. (1996). PchR, a regulator of ferripyochelin receptor gene $(f p t A)$ expression in Pseudomonas aeruginosa, functions both as an activator and as a repressor. J Bacteriol 178, 2586-2592.

Heurlier, K., Williams, F., Heeb, S., Dormond, C., Pessi, G., Singer, D., Camara, M., Williams, P. \& Haas, D. (2004). Positive control of swarming, rhamnolipid synthesis, and lipase production by the posttranscriptional RsmA/RsmZ system in Pseudomonas aeruginosa PAO1. J Bacteriol 186, 2936-2945.

Hunt, T. A., Peng, W. T., Loubens, I. \& Storey, D. G. (2002). The Pseudomonas aeruginosa alternative sigma factor $\mathrm{PvdS}$ controls exotoxin A expression and is expressed in lung infections associated with cystic fibrosis. Microbiology 148, 3183-3193.

Jacobs, M. A., Alwood, A., Thaipisuttikul, I. \& 12 other authors (2003). Comprehensive transposon mutant library of Pseudomonas aeruginosa. Proc Natl Acad Sci U S A 100, 14339-14344.

Juhas, M., Wiehlmann, L., Huber, B. \& 8 other authors (2004). Global regulation of quorum sensing and virulence by VqsR in Pseudomonas aeruginosa. Microbiology 150, 831-841.

Juhas, M., Eberl, L. \& Tummler, B. (2005). Quorum sensing: the power of cooperation in the world of Pseudomonas. Environ Microbiol 7, 459-471.

Klausen, M., Heydorn, A., Ragas, P., Lambertsen, L., AaesJorgensen, A., Molin, S. \& Tolker-Nielsen, T. (2003). Biofilm formation by Pseudomonas aeruginosa wild type, flagella and type IV pili mutants. Mol Microbiol 48, 1511-1524.

Kohler, T., van Delden, C., Curty, L. K., Hamzehpour, M. M. \& Pechere, J. C. (2001). Overexpression of the MexEF-OprN multidrug efflux system affects cell-to-cell signalling in Pseudomonas aeruginosa. J Bacteriol 183, 5213-5222.
Lamont, I. L., Beare, P. A., Ochsner, U., Vasil, A. I. \& Vasil, M. L. (2002). Siderophore-mediated signalling regulates virulence factor production in Pseudomonas aeruginosa. Proc Natl Acad Sci U S A 99, 7072-7077.

Laskowski, M. A., Osborn, E. \& Kazmierczak, B. I. (2004). A novel sensor kinase-response regulator hybrid regulates type III secretion and is required for virulence in Pseudomonas aeruginosa. Mol Microbiol 54, 1090-1103.

Liu, M. Y. \& Romeo, T. (1997). The global regulator CsrA of Escherichia coli is a specific mRNA-binding protein. J Bacteriol 179, 4639-4642.

Maseda, H., Saito, K., Nakajima, A. \& Nakae, T. (2000). Variation of the mexT gene, a regulator of the MexEF-oprN efflux pump expression in wild-type strains of Pseudomonas aeruginosa. FEMS Microbiol Lett 192, 107-112.

Mattick, J. S., Whitchurch, C. B. \& Alm, R. A. (1996). The molecular genetics of type-4 fimbriae in Pseudomonas aeruginosa - a review. Gene 179, 147-155.

Mavrodi, D. V., Bonsall, R. F., Delaney, S. M., Soule, M. J., Phillips, G. \& Thomashow, L. S. (2001). Functional analysis of genes for biosynthesis of pyocyanin and phenazine-1-carboxamide from Pseudomonas aeruginosa PAO1. J Bacteriol 183, 6454-6465.

McGrath, S., Wade, D. S. \& Pesci, E. C. (2004). Dueling quorum sensing systems in Pseudomonas aeruginosa control the production of the Pseudomonas quinolone signal (PQS). FEMS Microbiol Lett 230, 27-34.

McKnight, S. L., Iglewski, B. H. \& Pesci, E. C. (2000). The Pseudomonas quinolone signal regulates rhl quorum sensing in Pseudomonas aeruginosa. J Bacteriol 182, 2702-2708.

Pesci, E. C., Milbank, J. B., Pearson, J. P., McKnight, S., Kende, A. S., Greenberg, E. P. \& Iglewski, B. H. (1999). Quinolone signalling in the cell-to-cell communication system of Pseudomonas aeruginosa. Proc Natl Acad Sci U S A 96, 11229-11234.

Pessi, G. \& Haas, D. (2001). Dual control of hydrogen cyanide biosynthesis by the global activator GacA in Pseudomonas aeruginosa PAO1. FEMS Microbiol Lett 200, 73-78.

Pessi, G., Williams, F., Hindle, Z., Heurlier, K., Holden, M. T., Camara, M., Haas, D. \& Williams, P. (2001). The global posttranscriptional regulator RsmA modulates production of virulence determinants and $\mathrm{N}$-acylhomoserine lactones in Pseudomonas aeruginosa. J Bacteriol 183, 6676-6683.

Poole, K. (2001). Multidrug resistance in Gram-negative bacteria. Curr Opin Microbiol 4, 500-508.

Pruitt, B. A., Jr, McManus, A. T., Kim, S. H. \& Goodwin, C. W. (1998). Burn wound infections: current status. World J Surg 22, 135-145.

Rahme, L. G., Tan, M. W., Le, L., Wong, S. M., Tompkins, R. G., Calderwood, S. B. \& Ausubel, F. M. (1997). Use of model plant hosts to identify Pseudomonas aeruginosa virulence factors. Proc Natl Acad Sci U S A 94, 13245-13250.

Reimmann, C., Beyeler, M., Latifi, A., Winteler, H., Foglino, M., Lazdunski, A. \& Haas, D. (1997). The global activator GacA of Pseudomonas aeruginosa PAO positively controls the production of the autoinducer $N$-butyryl-homoserine lactone and the formation of the virulence factors pyocyanin, cyanide, and lipase. Mol Microbiol 24, 309-319.

Romeo, T. (1998). Global regulation by the small RNA-binding protein CsrA and the non-coding RNA molecule CsrB. Mol Microbiol 29, 1321-1330.

Sambrook, J., Fritsch, E. F. \& Maniatis, T. (1989). Molecular Cloning: a Laboratory Manual, 2nd edn. Cold Spring Harbor, NY: Cold Spring Harbor Laboratory. 
Savli, H., Karadenizli, A., Kolayli, F., Gundes, S., Ozbek, U. \& Vahaboglu, H. (2003). Expression stability of six housekeeping genes: a proposal for resistance gene quantification studies of Pseudomonas aeruginosa by real-time quantitative RT-PCR. J Med Microbiol 52, 403-408.

Schuster, M., Hawkins, A. C., Harwood, C. S. \& Greenberg, E. P. (2004). The Pseudomonas aeruginosa RpoS regulon and its relationship to quorum sensing. Mol Microbiol 51, 973-985.

Sobel, M. L., Neshat, S. \& Poole, K. (2005). Mutations in PA2491 (mexS) promote MexT-dependent mexEF-oprN expression and multidrug resistance in a clinical strain of Pseudomonas aeruginosa. J Bacteriol 187, 1246-1253.

Spaink, H. P., Okker, R. J. H., Wijffelman, C. A., Pees, E. \& Lugtenberg, B. J. J. (1987). Promoters in the nodulation region of the Rhizobium leguminosarum Sym plasmid pRL1J1. Plant Mol Biol 27-39.

Stintzi, A., Evans, K., Meyer, J. M. \& Poole, K. (1998). Quorumsensing and siderophore biosynthesis in Pseudomonas aeruginosa: lasR/lasI mutants exhibit reduced pyoverdine biosynthesis. FEMS Microbiol Lett 166, 341-345.

Toutain, C. M., Zegans, M. E. \& O'Toole, G. A. (2005). Evidence for two flagellar stators and their role in the motility of Pseudomonas aeruginosa. J Bacteriol 187, 771-777.

Vallet, I., Olson, J. W., Lory, S., Lazdunski, A. \& Filloux, A. (2001). The chaperone/usher pathways of Pseudomonas aeruginosa: identification of fimbrial gene clusters (сир) and their involvement in biofilm formation. Proc Natl Acad Sci U S A 98, 6911-6916.
Vallet, I., Diggle, S. P., Stacey, R. E., Camara, M., Ventre, I., Lory, S., Lazdunski, A., Williams, P. \& Filloux, A. (2004). Biofilm formation in Pseudomonas aeruginosa: fimbrial cup gene clusters are controlled by the transcriptional regulator MvaT. J Bacteriol 186, 2880-2890.

Van Delden, C. \& Iglewski, B. H. (1998). Cell-to-cell signalling and Pseudomonas aeruginosa infections. Emerg Infect Dis 4, 551-560.

Visca, P., Leoni, L., Wilson, M. J. \& Lamont, I. L. (2002). Iron transport and regulation, cell signalling and genomics: lessons from Escherichia coli and Pseudomonas. Mol Microbiol 45, 1177-1190.

Wade, D. S., Calfee, M. W., Rocha, E. R., Ling, E. A., Engstrom, E., Coleman, J. P. \& Pesci, E. C. (2005). Regulation of Pseudomonas quinolone signal synthesis in Pseudomonas aeruginosa. J Bacteriol 187, 4372-4380.

Wei, B. L., Brun-Zinkernagel, A. M., Simecka, J. W., Pruss, B. M., Babitzke, P. \& Romeo, T. (2001). Positive regulation of motility and flhDC expression by the RNA-binding protein CsrA of Escherichia coli. Mol Microbiol 40, 245-256.

West, S. E., Sample, A. K. \& Runyen-Janecky, L. J. (1994). The $v f r$ gene product, required for Pseudomonas aeruginosa exotoxin A and protease production, belongs to the cyclic AMP receptor protein family. J Bacteriol 176, 7532-7542.

Whiteley, M., Lee, K. M. \& Greenberg, E. P. (1999). Identification of genes controlled by quorum sensing in Pseudomonas aeruginosa. Proc Natl Acad Sci U S A 96, 13904-13909.

Wolfgang, M. C., Lee, V. T., Gilmore, M. E. \& Lory, S. (2003). Coordinate regulation of bacterial virulence genes by a novel adenylate cyclase-dependent signalling pathway. Dev Cell 4, 253-263. 